\title{
Digital Fluxgate Magnetometer for Detection of Microvibration
}

\author{
Menghui Zhi, ${ }^{1}$ Liang Tang, ${ }^{2}$ Xin Cao, ${ }^{3}$ and Donghai Qiao ${ }^{1}$ \\ ${ }^{1}$ School of Electronic and Information Engineering, Soochow University, 333 Ganjiang East Road, Suzhou 215006, China \\ ${ }^{2}$ Institute of Acoustics, Chinese Academy of Sciences, Beijing 100190, China \\ ${ }^{3}$ Institute Geology and Geophysics, Chinese Academy of Sciences, Beijing 100029, China \\ Correspondence should be addressed to Menghui Zhi; zhimenghui@yeah.net
}

Received 29 August 2016; Revised 15 November 2016; Accepted 1 December 2016; Published 5 January 2017

Academic Editor: Stefania Campopiano

Copyright (C) 2017 Menghui Zhi et al. This is an open access article distributed under the Creative Commons Attribution License, which permits unrestricted use, distribution, and reproduction in any medium, provided the original work is properly cited.

\begin{abstract}
In engineering practice, instruments, such as accelerometer and laser interferometer, are widely used in vibration measurement of structural parts. A method for using a triaxial fluxgate magnetometer as a microvibration sensor to measure low-frequency pendulum microvibration (not translational vibration) is proposed in this paper, so as to detect vibration from low-frequency vibration sources, such as large rotating machine, large engineering structure, earthquake, and microtremor. This method provides vibration detection based on the environmental magnetic field signal to avoid increased measurement difficulty and error due to different relative positions of permanent magnet and magnetometer on the device under test (DUT) when using the original magnetic measurement method. After fixedly connecting the fluxgate probe with the DUT during the test, the angular displacement due to vibration can be deduced by measuring the geomagnetic field's magnetic induction intensity change on the orthogonal three components during the vibration. The test shows that the microvibration sensor has angular resolution of over $0.05^{\circ}$ and maximum measuring frequency of $64 \mathrm{~Hz}$. As an exploring test aimed to detect the microvibration of earth-orbiting satellite in the in-orbit process, the simulation experiment successfully provides the real-time microvibration information for attitude and orbit control subsystem.
\end{abstract}

\section{Introduction}

Vibration is a common phenomenon in nature and can be divided into deterministic vibration and random vibration, whose monitoring and analysis technology plays an important role in engineering application and scientific measurement [1]. Microvibration generally refers to a low$g$ vibration generated when the structural body is subject to environmental change, external impact, and other structure movements [2]. In the field of engineering vibration test, there are mechanical, optical, and electric measuring methods based on physical properties during the test [3-5]. Taking Geiger mechanical vibrometer [6] as an example, in the mechanical measuring method, the parameter variation information of engineering vibration is first converted into the mechanical signal, which then is amplified and recorded with the mechanical system; this method has the advantage of easy operation, but it also has the disadvantage of poor measurement accuracy. In the optical measuring method, the parameter variation information of engineering vibration is first converted into the optical signal, which then is amplified and recorded with the subsequent optical system; this method has a higher accuracy but requires higher cost and more other devices compared with the mechanical measuring method, and the fiber optic microvibration sensor [7] is a typical representative of that. In the electric measuring method, the parameter variation information of engineering vibration is first converted into the electric signal, which then is amplified and recorded with built-in electronic circuits; this method features simple design, low cost, and good reliability, which is the most recognized and most widely used measuring method at present. For example, the frequently used seismic wave detector [8] and accelerometer [9] and so on conduct the measurement based on this method.

Based on the electric measuring method, some scholars at home and abroad are dedicated to the research on microvibration detection with the magnetic method. Djamal et al. $[10,11]$ conducted research on detecting the vibration of a 


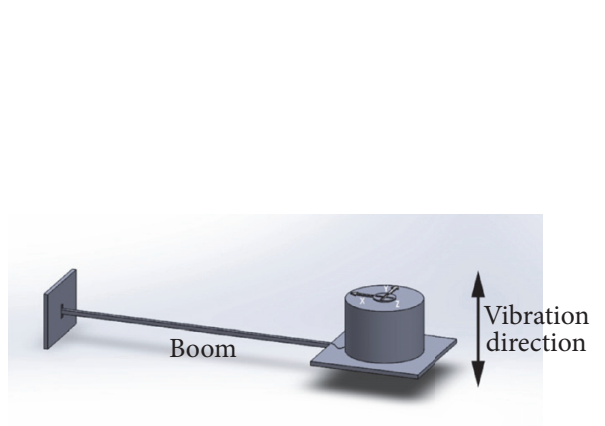

(a)

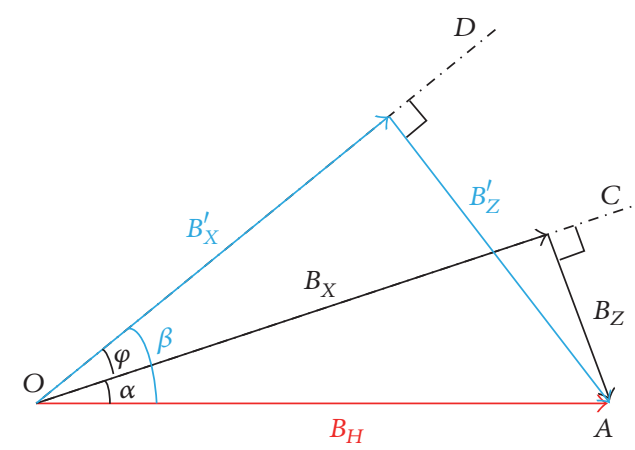

(b)

FIGURE 1: Microvibration detection: (a) physical model and (b) simplified model.

cantilever beam (with a permanent magnet at the end) with a simulating fluxgate component; Ren et al. [12] measured the vibration of the DUT connected to permanent magnet with a high-precision magnetometer, and this method obtains the angular displacement and linear displacement of microvibration from the model based on the change in magnetic induction intensity distribution of the permanent magnet caused by vibration. The ferromagnetic permanent magnet is used in both methods, but the distance between permanent magnet and magnetic field measuring equipment has a direct effect on the resolution of angle measurement, and the vibration model varies with the relative position between magnetic field measuring equipment and permanent magnet, which greatly increases the measurement difficulty and uncertainty of microvibration. In addition, external magnetic field shielding and reference field adding method is also a standard method in measuring vibration, which has a corresponding application in the equipment like seismic sensor [13], but merely in the general vibration-measuring field. External magnetic field shielding and reference field adding method is difficult to implement when measuring vibration of large-scale objects. Also, fluctuation of the added reference magnetic field might be larger than the periodic variation of the geomagnetic field (dozens of nanoteslas).

In view of these problems and the specific application of satellite microvibration measurement $[14,15]$, this paper proposes a method of deducing the angular displacement based on the geomagnetic field's magnetic induction intensity change on the orthogonal three components of the probe during the vibration measurement after fixedly connecting the probe of the triaxial digital fluxgate sensor with the DUT. This method is able to ensure a real-time measurement of spatial pendulum vibration. Starting from the principle of measuring microvibration with the fluxgate magnetometer, this paper introduces the operating principle of the fluxgate magnetometer, design of the magnetometer probe, and circuit board as well as final vibration test and proves the rationality and accuracy of its microvibration measurement.

\section{Detection Principle of Microvibration}

As shown in Figure 1(a), after installing the probe of the triaxial digital fluxgate magnetometer on the end of the horizontal boom, the magnetic induction intensity change of geomagnetic field on the probe three components due to vibration of the boom can be recorded with the magnetometer, and a relation can be established between change in the three-component magnetic induction intensity and vibration angle of the boom with the detection model, with which the angular displacement of the boom itself can be deduced. For convenience, only the vibration model in vertical direction is established, and the ones in other two directions can be obtained by analogy.

The fluxgate magnetometer is a high sensitive magnetic field vector detector, which can measure the magnetic induction intensity component in $X, Y$, and $Z$ direction, respectively, [16-18]. As shown in Figure 1(b), in the magnetic field coordinate system (the axis along the boom direction is $X$, the axis horizontally perpendicular to the boom direction is $Y$, and the axis perpendicular to $X O Y$ plane is $Z$; the three axes follow the right-hand rule), the environmental geomagnetic field's magnetic induction intensity vector component on $X O Z$ plane is $\mathbf{B}_{\mathrm{H}}$. Assume that the initial position of the boom is $O C$ (not perpendicular to $\mathbf{B}_{\mathbf{H}}$, i.e., $B_{X} \neq 0$ ), the magnetic induction intensity in $X$ and $Z$ direction measured with the magnetometer is $B_{X}$ and $B_{Z}$, respectively, and the angle $\alpha$ between boom and $\mathbf{B}_{\mathbf{H}}$ can be expressed as

$$
\alpha=\arctan \frac{B_{Z}}{B_{X}} .
$$

After microvibration, the vibration position of the boom is $O D$ (not perpendicular to $\mathbf{B}_{\mathbf{H}}$, i.e., $B_{X}^{\prime} \neq 0$ ), the magnetic induction intensity in $X$ and $Z$ direction measured with the magnetometer is $B_{X}^{\prime}$ and $B_{Z}^{\prime}$, and the angle $\beta$ between boom and $\mathbf{B}_{\mathbf{H}}$ can be expressed as

$$
\beta=\arctan \frac{B_{Z}^{\prime}}{B_{X}^{\prime}} .
$$

The angular displacement of the boom before and after the vibration is

$$
\varphi=\beta-\alpha
$$

This vibration detection model is applicable to vibration detection of massive low-frequency vibration sources in 
engineering practice as it relies on the geomagnetic field itself. However, the geomagnetic field was easier to be intervened by the external environment and the intervention of the external environment cannot be excluded in a single probe even if the designed fluxgate magnetometer has been calibrated (reducing measuring errors of the instrument). On this occasion, multiple probes (generally 2 or 3 probes, one is placed on the measured parts and another one or two are placed in the relative prohibited environment of the same space) can be utilized together on this occasion. Influences brought by external intervention and geomagnetic field changes can be excluded from this measured method. That is, the displacement information and angle information of vibration can be deduced by extracting differential mode signal to detect the actual three-component periodic variation of magnetic induction strength influenced by microvibration. As the model is based on the magnetic field measurement technology of high-precision triaxial digital fluxgate magnetometer, the design of the magnetometer is essential in the detection method of this microvibration.

\section{Design of Fluxgate Magnetometer}

The fluxgate magnetometer is an improved transformer device, which mainly consists of fluxgate probe and fluxgate signal processing circuit. The alternation of periodic saturation and unsaturation is formed on the magnetic core under the common action of external magnetic field and periodic alternating excitation magnetic field (the frequency is set to be $f_{0}$ ), and the pick-up coils around the magnetic core will generate pick-up signals containing the magnetic field information; that is, the fluxgate probe will convert environmental magnetic field signals into electric signals. At this point, pick-up signals contain various harmonic components where the even order harmonic contains the information about environmental magnetic field; as the second harmonic has the maximum amplitude in even order harmonics, the fluxgate signal processing circuit that corresponds to the second harmonic method is used to extract the information about environmental magnetic field. The system adopts closed-loop structure to ensure the overall linearity, measurement range, resolution, and reliability.

3.1. Fluxgate Probe. The fluxgate probe mainly consists of magnetic core, excitation coils, pick-up coils, feedback coils, and framework and chassis used to support magnetic core and coils. The probe's performance plays a decisive role in indexes of the fluxgate magnetometer; its structural model is shown in Figures 2(a) and 2(b). Two magnetic cores (with diameters of $13 \mathrm{~mm}$ and $18 \mathrm{~mm}$ respectively) with excitation coils are vertically nested to make them under the state of periodic oversaturation through the external excitation circuit and internal LC resonance (the red component in Figure 2(c) is the resonant capacitor). The pick-up coils are attached as close as possible to the ring cores to increase the signal to noise ratio of the system, while the outer Helmholtz coils (feedback coils) are used to compensate the external field at the ring core position. The vector compensation system keeps the sensitive probe in zero field, which fully guarantees the linearity of the equipment. The framework and chassis are designed to ensure the orthogonality between coils and reduce the interference between each other. Moreover, the combination of materials with different thermal expansion coefficients can be avoided by using the additional mechanical support. Figure 2(c) shows the physical map of fluxgate probe.

The selection of magnetic core is especially important for design, which will mainly consider the following factors $[19,20]$ : high magnetic permeability, low coercivity, low magnetostriction, low Barkhausen noise, low internal stress and structure defect, low saturation magnetization, high surface evenness, and high electrical resistivity. Based on the above principles, permalloy 1 1J86 is selected to produce the magnetic core, which has the noise level lower than $10 \mathrm{pT} \cdot \mathrm{Hz}^{-1 / 2}$ at $1 \mathrm{~Hz}$. Each component's pick-up signal containing the information about environmental magnetic field will be generated in the three-axis pick-up coils, and the three-axis feedback coils will be used to compensate the environmental magnetic field in the central position of the probe to make it approximate zero field.

3.2. Fluxgate Signal Processing Circuit. The functional block diagram of fluxgate signal processing circuit and picture of circuit board are shown in Figure 3, which mainly include field programmable gate array (FPGA) control unit, excitation circuit, detection circuit, feedback circuit, and interface control circuit.

Its operating principle is as follows: as shown in Figure $4(\mathrm{a})$, the square signal (with the frequency $f_{0}$ of $9.6 \mathrm{kHz}$ ) generated by FPGA is amplified by the power amplifier and LC resonance network to apply the resulting alternating current to the excitation coils and drive the magnetic core into the positive or negative saturation state, and the positive flux of the magnetic core is increased while the negative flux is decreased in the environmental magnetic field, resulting in unbalance flux in the magnetic core and obtaining the excitation current waveform as shown in Figure 4(b). For each excitation current reversal, pick-up signals that rely on the environmental magnetic field amplitude and polarity exist in the pick-up coils, which are all even order harmonics. At this point, the detection circuit (used to detect second harmonic) extracts the second harmonic component with the LC resonance network (with resonance frequency of $2 f_{0}$ ) and difference preamplifier, as shown in Figure 3. Different from the traditional fluxgate magnetometer that realizes the backend phase-sensitive rectification and smooth filtering and so on with the analog circuit, this design samples amplified signals with the fully differential ADC directly (at sampling frequency of $4 f_{0}$ ) and sends them into FPGA for processing. By completing various functions of data processing, FPGA effectively reduces the complexity of circuit structure and improves the resistance against external interference. Finally, the calculated compensation voltage is converted into the corresponding compensation current through the feedback circuit as shown in Figure 5, and the reversing field that compensates the environmental magnetic field is generated 


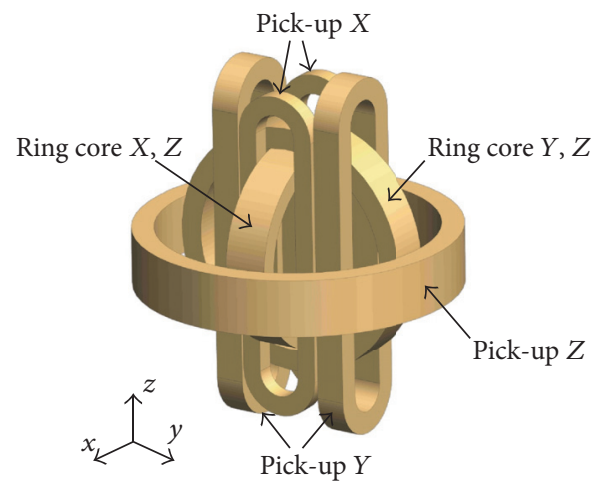

(a)

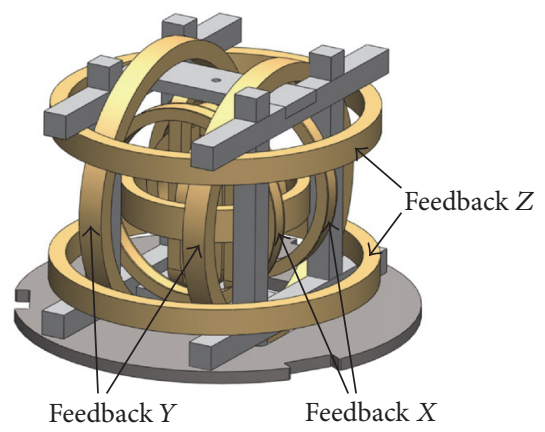

(b)

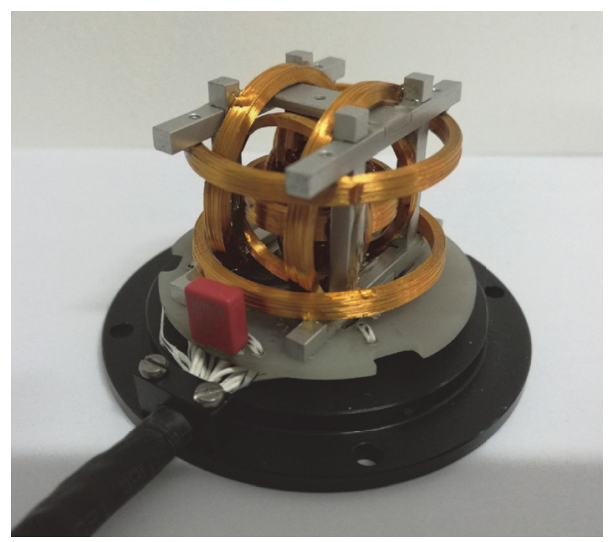

(c)

FIGURE 2: 3D model with (a) ring cores and pick-up coil system, (b) the whole structure including the Helmholtz feedback system, and (c) physical map of fluxgate probe.

in the feedback coils. As the magnetic induction intensity in the final position of magnetic core is approximately zero, the magnetic induction intensity in the current environment can be obtained through the actual reversal compensation of magnetic induction intensity. The interface control circuit is designed to assist in completing the remote command control of the magnetometer, synchronizing with GPS and transferring the magnetic field data, and realize remote measurement of magnetic field under adverse environmental conditions.

In order to ensure full coverage and high-accuracy measurement of the geomagnetic field, a triaxial fluxgate magnetometer with linear dynamic range of $\pm 65000 \mathrm{nT}$ and resolution of $0.02 \mathrm{nT}$ is designed, and its feedback circuit is shown in Figure 5.

According to the manufacturer's instructions of LTC1595 $[21,22]$, the compensation current in the $x$-axis Helmholtz coils can be expressed as

$$
\begin{array}{r}
i_{c}=7.29 \times 10^{-3} k_{1}^{\prime}+7.88 \times 10^{-5} k_{2}^{\prime}-3.64 \times 10^{-3}, \\
0 \leq k_{1}^{\prime} \leq 1,0 \leq k_{2}^{\prime} \leq 1 .
\end{array}
$$

The range of compensation current is from $-3.64 \mathrm{~mA}$ to $3.73 \mathrm{~mA}$, which basically meets the symmetric relation.
Feedback coils are of Helmholtz structure [23], and the magnetic induction intensity at the midpoint $O$ is

$$
B_{0}=\frac{8 \mu_{0} N I}{5 \sqrt{5} R}
$$

where $\mu_{0}$ is vacuum permeability, $N$ is turns per currentcarrying coil, $I$ is excitation current intensity of coils, and $R$ is average radius of coils. Taking Helmholtz coils in $X$ direction as an example, a single current-carrying coil with the average radius $R$ of $12 \mathrm{~mm}$ and turns $N$ of 280 is wound. As the vacuum permeability (approximate to air permeability) is $\mu_{0}=4 \pi \times 10^{-7} \mathrm{~N} / \mathrm{A}^{2}$, the magnetic induction intensity in the central position of the probe can be expressed as

$$
\begin{array}{r}
B_{\mathrm{OX}}=1.53 \times 10^{-4} k_{1}^{\prime}+1.65 \times 10^{-6} k_{2}^{\prime}-7.64 \times 10^{-5}, \\
0 \leq k_{1}^{\prime} \leq 1,0 \leq k_{2}^{\prime} \leq 1 .
\end{array}
$$

The range of compensation field generated in feedback coils is from $-76400 \mathrm{nT}$ to $78300 \mathrm{nT}$; that is, the magnetic field compensation capability generated by feedback coils in $X$ direction can cover the scope of geomagnetic field ( $\pm 65000 \mathrm{nT}$ ). By using the similar method, magnetic induction intensity expressions for $Y(r=649 \Omega, R=15 \mathrm{~mm}$, and 


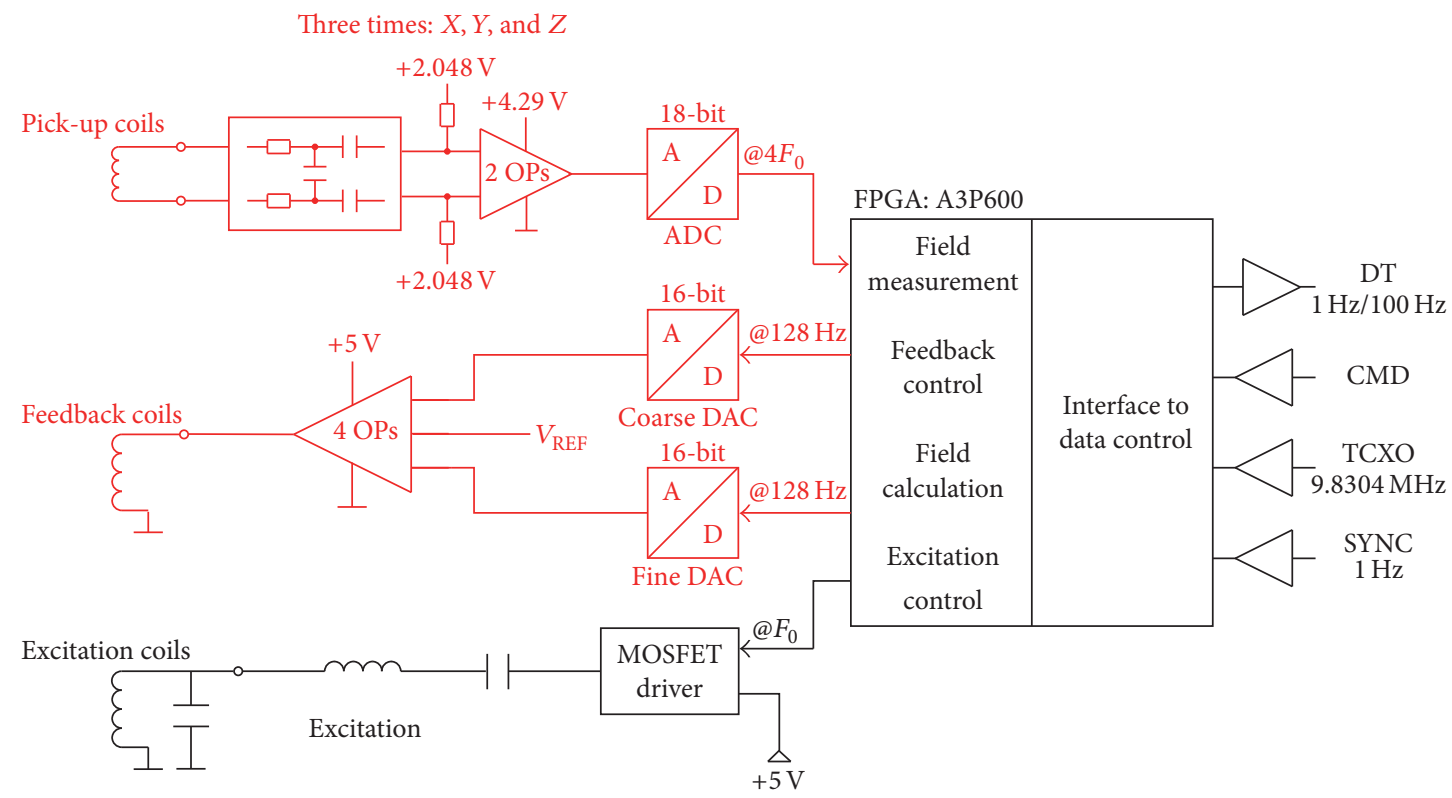

(a)

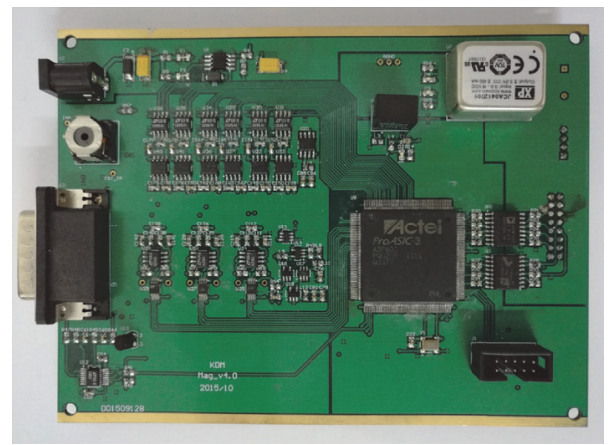

(b)

FIGURE 3: (a) Functional block diagram and (b) printed circuit board of fluxgate signal processing circuit.

$N=270)$ and $Z$ direction $(r=536 \Omega, R=17.5 \mathrm{~mm}$, and $N=230)$ are

$$
\begin{array}{r}
B_{\mathrm{OY}}=1.50 \times 10^{-4} k_{1}^{\prime}+1.62 \times 10^{-6} k_{2}^{\prime}-7.48 \times 10^{-5}, \\
0 \leq k_{1}^{\prime} \leq 1,0 \leq k_{2}^{\prime} \leq 1, \\
B_{\mathrm{OZ}}=1.33 \times 10^{-4} k_{1}^{\prime}+1.43 \times 10^{-6} k_{2}^{\prime}-6.61 \times 10^{-5}, \\
0 \leq k_{1}^{\prime} \leq 1,0 \leq k_{2}^{\prime} \leq 1 .
\end{array}
$$

We can get the following result: the range of compensation field generated by $y$ - and $z$-axis is from $-74800 \mathrm{nT}$ to $76800 \mathrm{nT}$ and $-66100 \mathrm{nT}$ to $68300 \mathrm{nT}$, respectively, which also satisfy the requirements for dynamic range; that is, the three-axis feedback loop designed is able to realize full compensation of the environmental magnetic field. At this point, the magnetic core position is under zero magnetic field or approximate zero magnetic field (known as linear zone, i.e., the zone where the ratio $k$ of magnetic field variation to 18-bit ADC sampling numerical variation is constant) state. When the control voltage is beyond the linear zone, FPGA control unit will perform search approaching again based on PID algorithm, until the magnetic core position reforms zero magnetic field or enters into the linear zone. After being stabilized, the output signal of the fluxgate probe represents the difference $[24,25]$ between current magnetic induction intensity $B(t)$ and feedback magnetic induction intensity $B(t-1)$ (the feedback magnetic induction intensity equals the last magnetic induction intensity but with the opposite direction); that is, the current magnetic induction intensity can be expressed as

$$
B(t)=B(t-1)+k A,
$$

where $A$ is the current sampling value of the difference ADC, which can be positive or negative.

Among all modules of the fluxgate signal processing circuit, FPGA control unit is an essential part, which can be used to control the excitation signal, measure the magnetic field signal, calculate the compensation current, control the feedback signal, and process the interface signal, and its functional block diagram is shown in Figure 6. To solve 


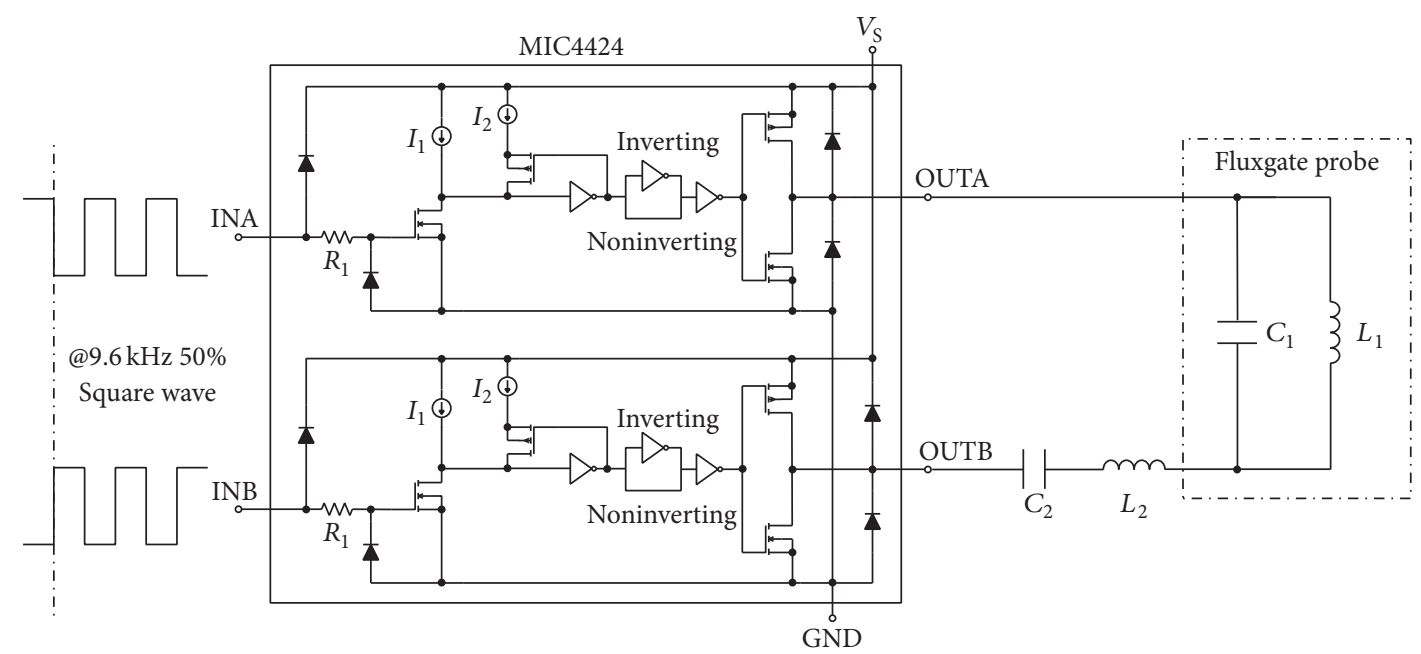

(a)

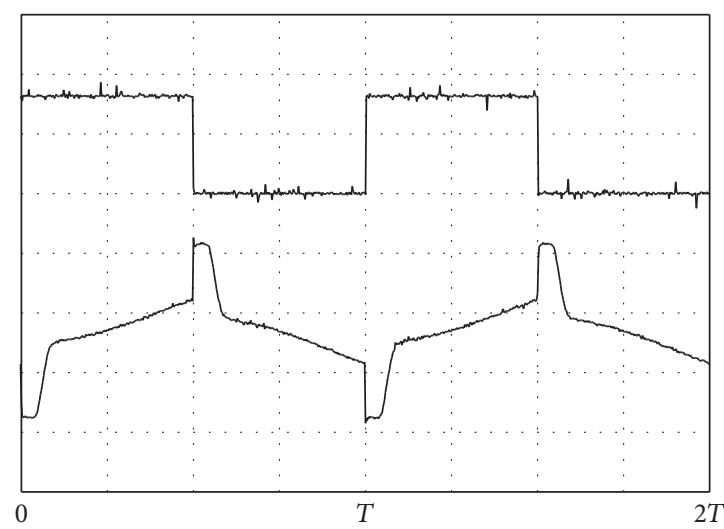

(b)

FIGURE 4: The (a) push-pull driver circuit and (b) excitation waveform and current in excitation coils.

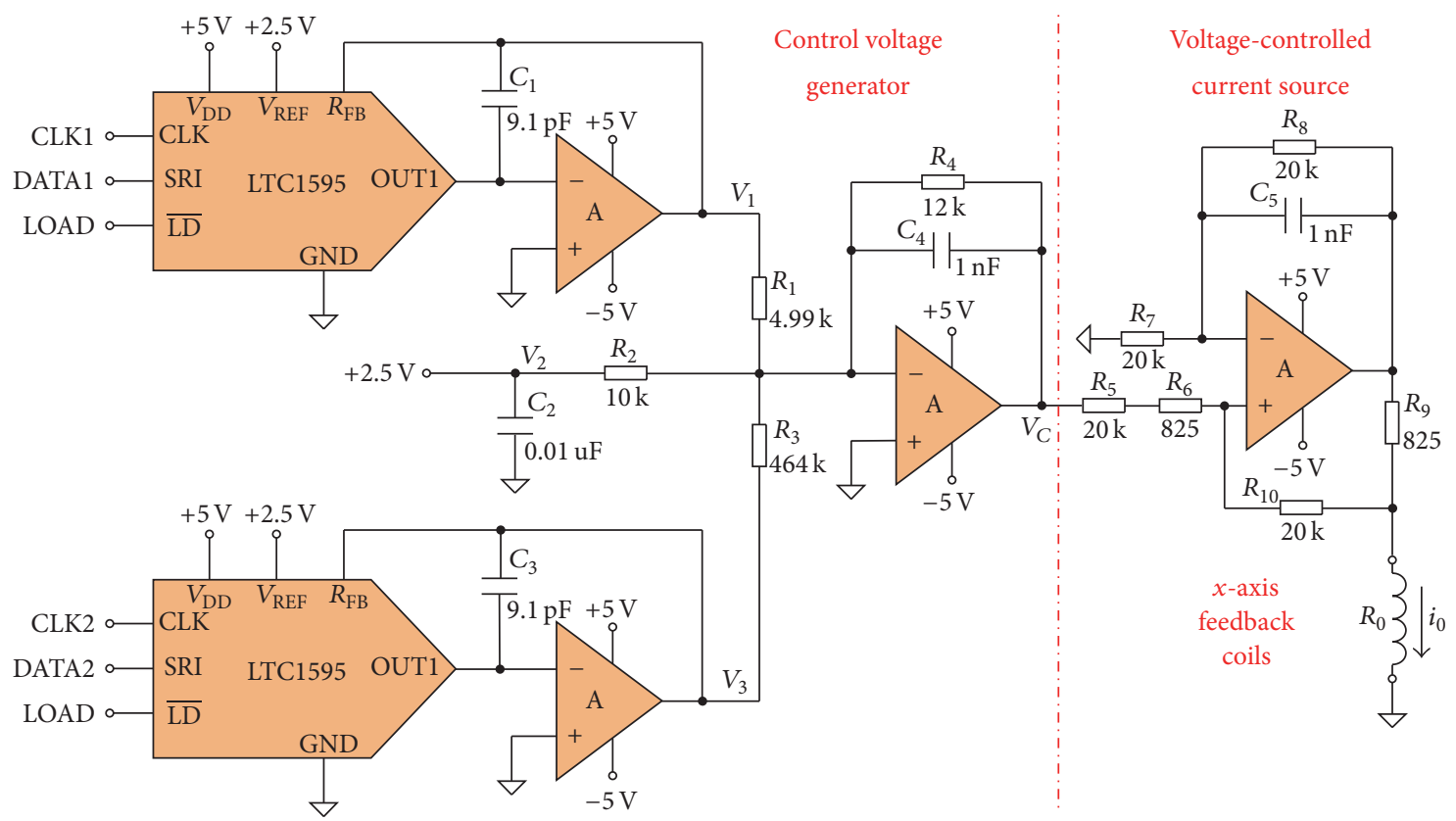

FIGURE 5: The feedback circuit. 


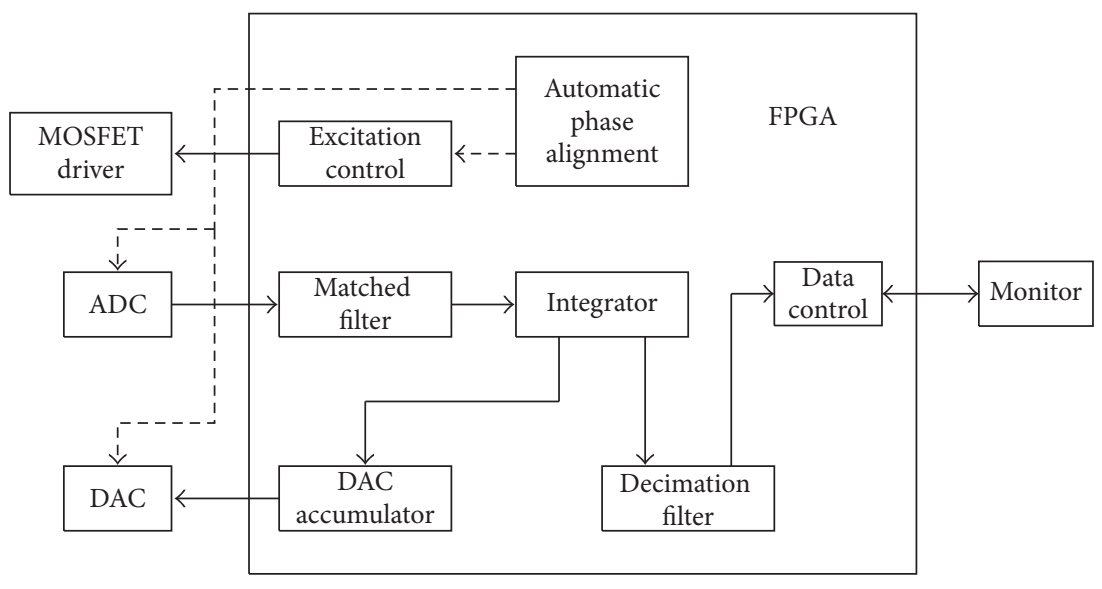

FIGURE 6: Functional block diagram of FPGA.

the problem of reduced digital filter performance due to the inconsistency between phase delay and axial delay of signal processing circuit of the triaxial fluxgate magnetometer, an automatic phase alignment module is used in FPGA to achieve the optimal data processing effect. In addition, the design of a decimation filter [26] is of great importance during the data processing of magnetic field, which must meet the requirements of flatness in band and rapid attenuation outside band to the greatest extent. Therefore, the cascade mode of the three-stage digital filter is adopted with the first stage of CIC filter and the second and third stage of FIR filter. The CIC filter is a class of hardware-efficient linear phase finite impulse response digital filters. CIC filters achieve sampling rate decimation without using multipliers, so they are widely deployed as the first stage of decimation filters. A FIFO buffer FIFO1 is used to bridge the CIC filter and FIR1 filter. The FIR1 filter stage has decimation by four-FIR filter structure. It compensates for the CIC filter droop and flattens the magnitude response of the pass band. Another FIFO buffer FIFO2 is used to bridge the FIR1 filter and FIR2 filter. The FIR2 filter stage has decimation by two-FIR filter structure. It creates a low-pass brick wall filter to block all the aliased components from downsampling.

\section{Experiment and Analysis}

4.1. Test of Fluxgate Magnetometer. To test the performance of the designed fluxgate magnetometer, a field contrast experimental facility, as shown in Figure 7, was built in Xishan, Suzhou (east longitude: $120^{\circ} 16^{\prime} 37^{\prime \prime}$; north latitude: $31^{\circ} 7^{\prime} 18^{\prime \prime}$; elevation: $130 \mathrm{~m}$ ) on April 26, 2016. The left and right systems in Figure 7(b) are fluxgate magnetometer designed in this paper and Magson standard fluxgate magnetometer made in Germany, respectively. Both consist of power supply, fluxgate probe, and fluxgate electronics box, where the supply provides power to the whole system, and the electronics box includes fluxgate signal processing circuit board and data storage circuit board to cooperate with the fluxgate probe for detection and real-time storage of magnetic field signal. In order to better describe the performance of the magnetometer designed, three axes of two probes shall be parallel to each other.

Geomagnetic field measurement of the designed magnetometer (KDM) and standard magnetometer (Magson) (red, Magson; black, KDM; and blue, difference value) performed in north-south direction of the Earth field can be seen in Figure 8(a). The Magson magnetometer, usually applied in geomagnetic observatories, can be used as standard because it is well tested and verified on many occasions. Figure 8(a) shows that the field variations measured with the designed magnetometer well confirm the result of the standard magnetometer, and the measurement error is less than $\pm 0.1 \mathrm{nT}$. Additionally, noise level of fluxgate magnetometer is mainly decided by background noise of probe and electronics unit, directly affecting accuracy of the magnetometer. The noise level of the same magnetometer is $7.393 \mathrm{pT} \cdot \mathrm{Hz}^{-1 / 2}$ at $1 \mathrm{~Hz}$ by placing the fluxgate probe in the shield cylinder, as shown in Figure $8(\mathrm{~b})$. And the statistics data of six designed fluxgate magnetometers in the same batch are all around $7 \mathrm{pT} \cdot \mathrm{Hz}^{-1 / 2}$ at $1 \mathrm{~Hz}$ that reflects the basic noise level of the designed magnetometers. The fluxgate magnetometer designed was sent to the Environmental Laboratory of China Academy of Space Technology for field test, which showed that measurement ranges of three axes of the magnetometer met the requirement of $\pm 65000 \mathrm{nT}$ with a good linearity. In other words, the designed magnetometer can satisfy the requirements of geomagnetic field measurement.

4.2. Microvibration Test. The microvibration test system built is shown in Figure 9(a). A $2.3 \mathrm{~m}$ boom with a fluxgate probe installed at the end is fixed on the three-axle table, and the electronics box is fixed at the center of the table and connected to the probe with cables. The three-axle table has microvibration with amplitude of $0.05^{\circ}$ and frequency of $0.25 \mathrm{~Hz}$ in vertical direction while rotating at angular velocity of $90^{\circ} / \mathrm{s}$. Three-circle measured data is selected for analysis; Figures 9(b) and 9(c) give the power spectrum chart and dynamic spectrum of vibration, respectively. As shown in Figure 9(b), the device can monitor the vibration signal of no more than $64 \mathrm{~Hz}$, which matches with the magnetic 


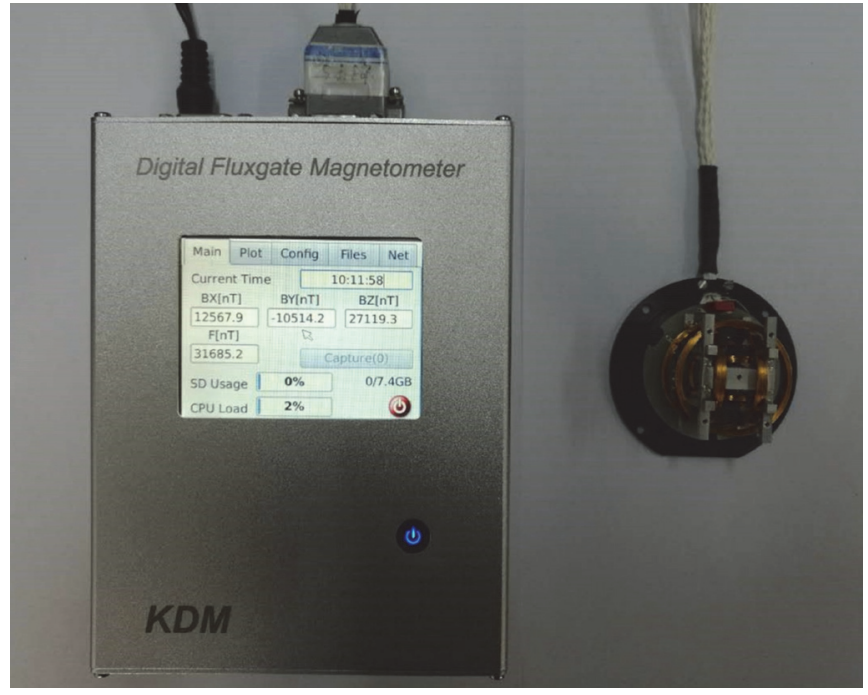

(a)

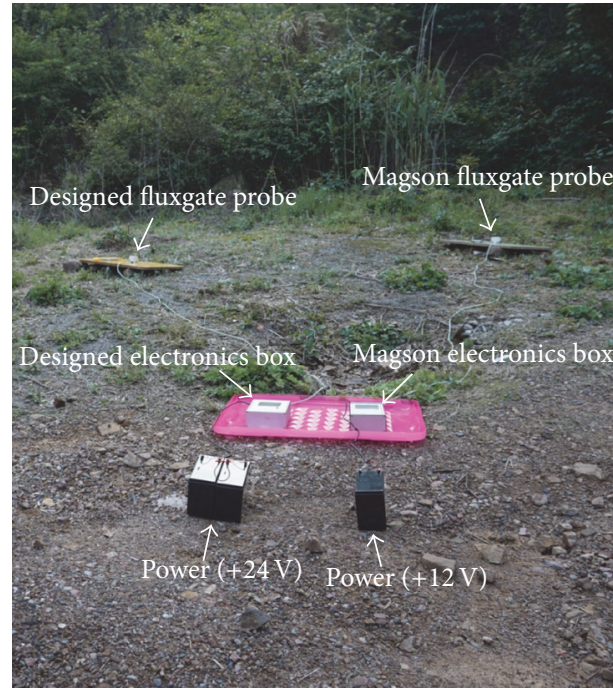

(b)

FIgURE 7: The (a) designed fluxgate magnetometer and (b) comparative experiment with Magson standard fluxgate magnetometer.

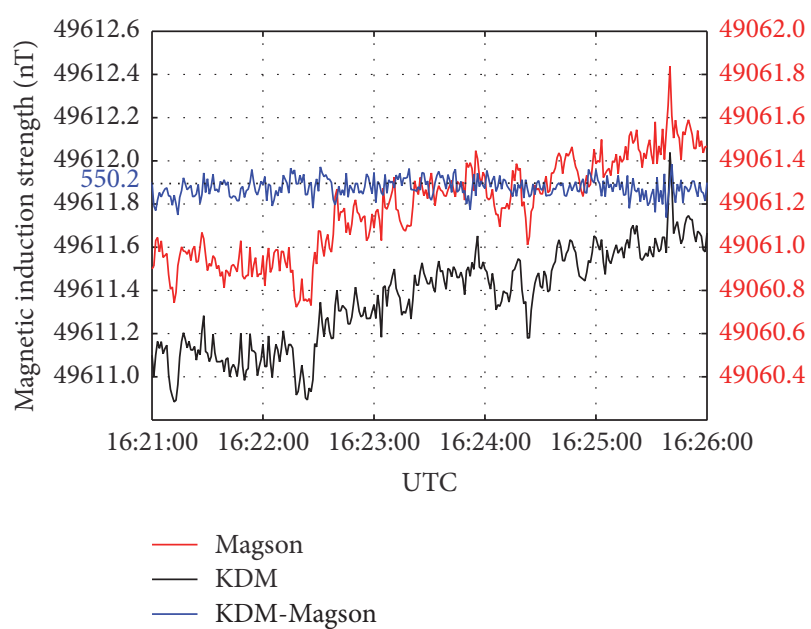

(a)

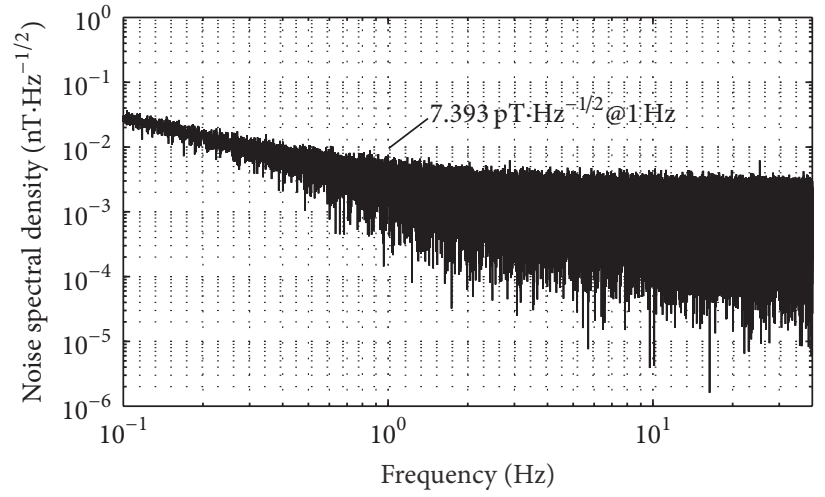

(b)

FIgURE 8: (a) Comparative curves of geomagnetic field. (b) Noise spectral density of the designed magnetometer.

field sampling frequency of $128 \mathrm{~Hz}$ designed in the fluxgate magnetometer. In addition to $0.25 \mathrm{~Hz}$ fundamental frequency for horizontal rotation of the table, many harmonic components with decreasing energy exist. However, the energy of peak around $5 \mathrm{~Hz}$ is significantly higher than the one of surrounding frequencies, and this frequency corresponds to the high-frequency disturbance in the test result. If this high-frequency disturbance is caused by the change in spatial position, this disturbance in each circle does not necessarily persist, and disturbances in different circles will occur in the same position; on the contrary, if this disturbance is caused by the boom itself, it will always exist and different circles do not necessarily have the same disturbance waveform. Therefore, wavelet analysis is conducted on the data, and various harmonic components are filtered to obtain the dynamic spectrogram as shown in Figure 9(c). The horizontal axis is number of points collected, that is, time while the vertical axis is frequency; the color in the figure represents the power spectral density of vibration signal, that is, vibration intensity. As can be seen in the figure, the disturbance persists, but disturbances in different circles do not have repeatability. Therefore, this high-frequency disturbance is initially determined to be the result of resonance between high-frequency swing harmonic of table and natural frequency of boom. The magnetic field data is processed (including removing the environmental static field and reducing the environmental noise) to extract the data of change in magnetic induction intensity due to microvibration, and the data is converted to the deflection angle of the boom based on the detection principle of microvibration to obtain the curve of boom vibration angle as a function of time as shown in Figure 9(d). It can be seen that horizontally rotating one cycle collects 


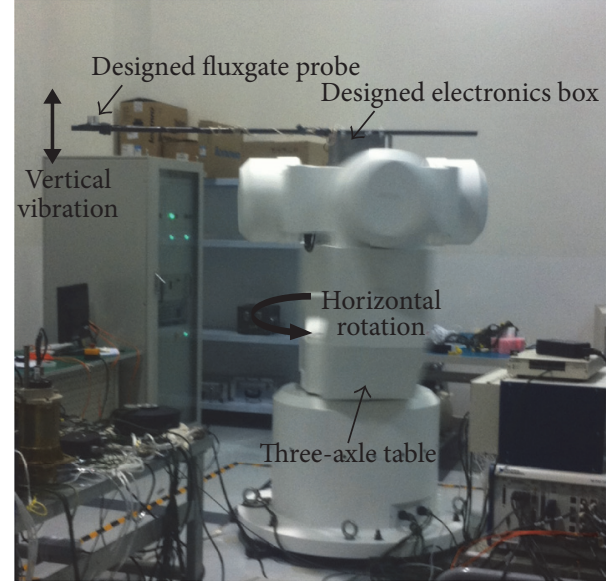

(a)

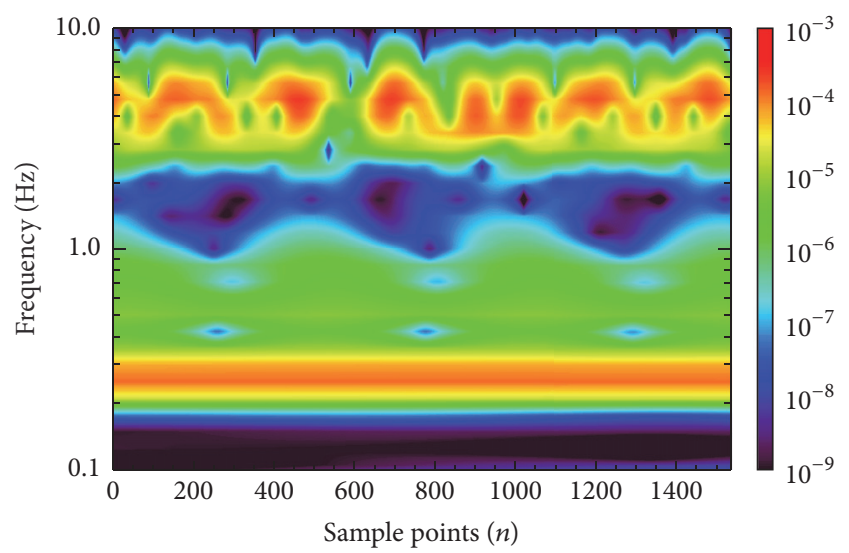

(c)

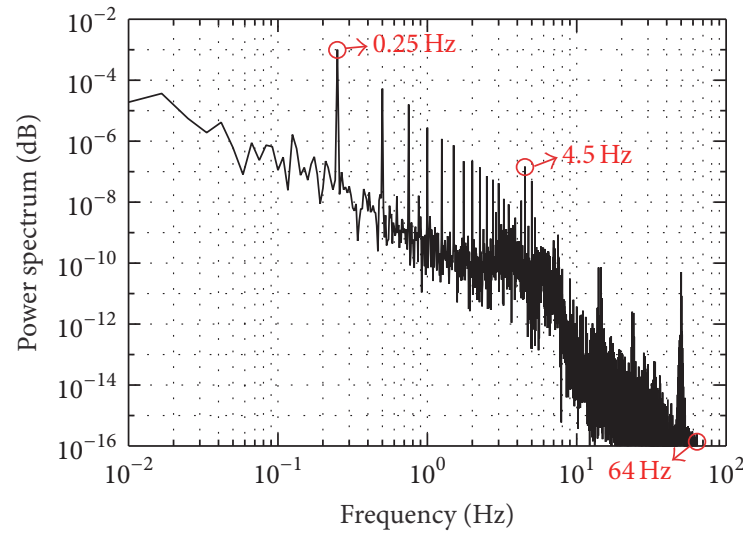

(b)

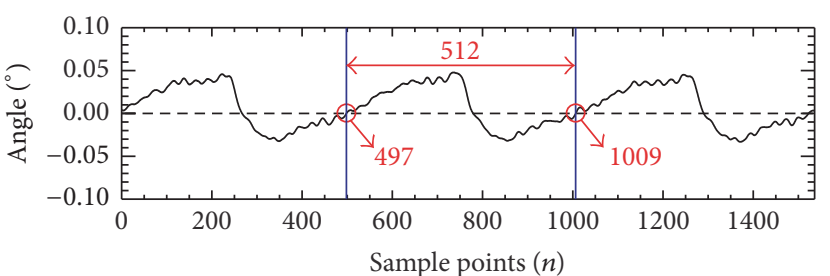

(d)

Figure 9: (a) Microvibration test system. (b) Power spectrum of vibration. (c) Dynamic spectrum of vibration. (d) Angular displacement of vertical vibration.

512 points, and the period of rotation obtained is $4 \mathrm{~s}$ based on the sampling frequency of $128 \mathrm{~Hz}$. The high-frequency disturbance information and angle variation of approx. $0.05^{\circ}$ can be obtained from the amplitude variation of the curve, truly reflecting the amplitude of microvibration in the vertical direction. In addition, the measuring accuracy of the equipment is superior to $0.01^{\circ}$, which can be verified by small vibration superposed on the angular displacement waveform of the vertical vibration shown in Figure 9(d).

\section{Conclusion}

In this paper, a method is proposed for detecting microvibration with a triaxial digital fluxgate magnetometer. This method allows the fluxgate magnetometer to be converted into the microvibration sensor to monitor the vibration of DUT in real time. In addition, this method used to measure microvibration is based on the relative change in geomagnetic component, which can effectively improve accuracy and anti-interference ability of the measurement system. The microvibration sensor designed with this method features high angular resolution, simple process circuit, fast frequency response, and so on, which provides a way of vibration detection for satellite attitude and orbit control subsystem.

Compared with the traditional magnetic measurement method, the environmental noise and noise performance of the magnetometer have a certain effect on microvibration measurement, and therefore the noise elimination technology can be further optimized in follow-up improvement. In addition, the high-frequency disturbance during the measurement can be further confirmed and identified by conducting the modal experiment on the boom or changing existing experiment parameters.

\section{Competing Interests}

The authors declare that there is no conflict of interests regarding the publication of this paper. 


\section{Acknowledgments}

This work is supported by National Science and Technology Major Project no. ZDYZ2012-1-01-02.

\section{References}

[1] H. Fein, "An application of holographic interferometry for dynamic vibration analysis of a jet engine turbine compressor rotor," in Proceedings of the Technologies for Synthetic Environments: Hardware-in-the-Loop Testing VIII, vol. 5092 of Proceedings of SPIE, pp. 307-315, Orlando, Fla, USA, April 2003.

[2] H. Miao, G. L. Song, and Q. Liu, "Micro-vibration analysis and measurement on high resolution remote sensing satellites," Applied Mechanics and Materials, vol. 232, pp. 432-436, 2012.

[3] T. Barszcz, "Proposal of a new method of mechanical vibration measurement," Metrology and Measurement Systems, no. 4, pp. 409-421, 2004.

[4] M. Ueda, T. Yamaguchi, H. Kakiuchi, and H. Suga, "A simple optical method for measuring the vibration amplitude of a speaker," Optics and Lasers in Engineering, vol. 32, no. 1, pp. 2128, 1999.

[5] Y. Q. Fu, "A marine engine torsion vibration measuring method and its implementation based on FPGA," in Proceedings of the IEEE Canadian Conference on Electrical and Computer Engineering (CCECE '02), vol. 1, pp. 488-493, Winnipeg, Canada, May 2002.

[6] Z. H. Zhang, T. Y. Zhang, Z. Q. Wang, and B. Q. Xu, "Electrical signal output from geiger vibrograp," Engineering Mechanics, vol. 3, no. 1, pp. 93-98, 1986.

[7] A. K. Ganguly and A. Saha, "Fiber-optic micro-displacement and vibration sensor," in Proceedings of the International Society for Optical Engineering, vol. 3541 of Proceedings of the SPIE, pp. 116-124, Boston, Mass, USA, November 1998.

[8] M. Gassenmeier, C. Sens-Schönfelder, T. Eulenfeld et al., "Field observations of seismic velocity changes caused by shakinginduced damage and healing due to mesoscopic nonlinearity," Geophysical Journal International, vol. 204, no. 3, pp. 1490-1502, 2016.

[9] Q. H. Fu, C. J. Li, and Y. F. Hou, "Vibration measurement of dental CBCT based on 3D accelerometer," in Proceedings of the IEEE International Conference on Medical Imaging Physics and Engineering (ICMIPE '13), pp. 97-101, Shenyang, China, October 2013.

[10] M. Djamal, Yulkifli, A. Setiadi, and R. N. Setiadi, "Development of a low cost vibration sensor based on fluxgate element," in Proceedings of the International Conference on Circuits, Systems, Signals (CSS '10), pp. 248-251, September 2010.

[11] M. Djamal, S. Ramli, and Suprijadi, "Development of a low cost vibration sensor based on flat coil element," International Journal of Mathematical Models and Methods in Applied Sciences, vol. 5, no. 3, pp. 550-557, 2011.

[12] Q. Y. Ren, H. Zhao, G. Q. Liu et al., "Method and system for micro-vibration with the aid of magnetic field measurement," CN CN103364073 A, 2013, (Chinese).

[13] T. Forbriger, R. Widmer-Schnidrig, E. Wielandt, M. Hayman, and N. Ackerley, "Magnetic field background variations can limit the resolution of seismic broad-band sensors," Geophysical Journal International, vol. 183, no. 1, pp. 303-312, 2010.

[14] S. Fan and L. Cao, "The development of micro-vibration for satellite," in Proceedings of the 8th International Symposium on Precision Engineering Measurements and Instrumentation, vol. 8759 of Proceedings of SPIE, pp. 311-313, Chengdu, China, August 2012.

[15] W. P. Li, H. Huang, X. B. Zhou, X. T. Zheng, and Y. Bai, "Design and experiments of an active isolator for satellite microvibration," Chinese Journal of Aeronautics, vol. 27, no. 6, pp. 1461-1468, 2014.

[16] W. Magnes, D. Pierce, A. Valavanoglou et al., "A sigma-delta fluxgate magnetometer for space applications," Measurement Science and Technology, vol. 14, no. 7, pp. 1003-1012, 2003.

[17] V. Korepanov and R. Berkman, "Digital flux-gate magnetometer structural analysis," Measurement Science and Technology, vol. 10, no. 8, pp. 734-737, 1999.

[18] A. Cerman and P. Ripka, "Towards fully digital magnetometer," Sensors and Actuators A: Physical, vol. 106, no. 1-3, pp. 34-37, 2003.

[19] Liangjintao and L. L. Wei, "Study on silicon-based microfluxgate permalloy core film," in Proceedings of the 4th IEEE Conference on Industrial Electronics and Applications (ICIEA '09), pp. 1196-1198, Xi'an, China, May 2009.

[20] O. Zorlu, P. Kejik, and R. S. Popovic, "An orthogonal fluxgatetype magnetic microsensor with electroplated Permalloy core," Sensors and Actuators A: Physical, vol. 135, no. 1, pp. 43-49, 2007.

[21] P. Bertemes-Filho, A. Felipe, and V. C. Vincence, "High accurate howland current source: output constraints analysis," Circuits and Systems, vol. 4, no. 7, pp. 451-458, 2013.

[22] E. L. Bronaugh, "Helmholtz coils for calibration of probes and sensors: limits of magnetic field accuracy and uniformity," in Proceedings of the IEEE International Symposium on Electromagnetic Compatibility, pp. 72-76, August 1995.

[23] S. Turner, M. J. Hall, S. A. C. Harmon, and N. Hillier, "Calibration of a novel three-axis fluxgate gradiometer for space applications," IEEE Transactions on Magnetics, vol. 51, no. 1, Article ID 4001704, 2015.

[24] W. Gao, G. C. Wang, and Q. C. Zhao, "Design and realization of fluxgate sensor signal processing unit," in Proceedings of the IEEE International Conference on Mechatronics and Automation, pp. 2386-2391, Beijing, China, August 2011.

[25] H. U. Auster, K. H. Glassmeier, W. Magnes et al., “The THEMIS fluxgate magnetometer," Space Science Reviews, vol. 141, no. 1-4, pp. 235-264, 2008.

[26] J. B. Wang and X. Chen, "A fluxgate magnetometer for navigation and sensing: noise character and digital filtering," in Proceedings of the IEEE Sensors, pp. 1-4, November 2015. 


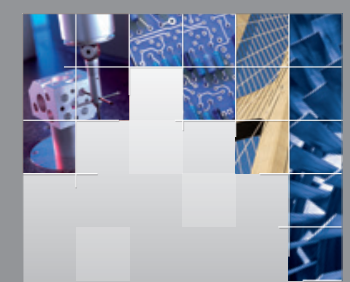

\section{Enfincering}
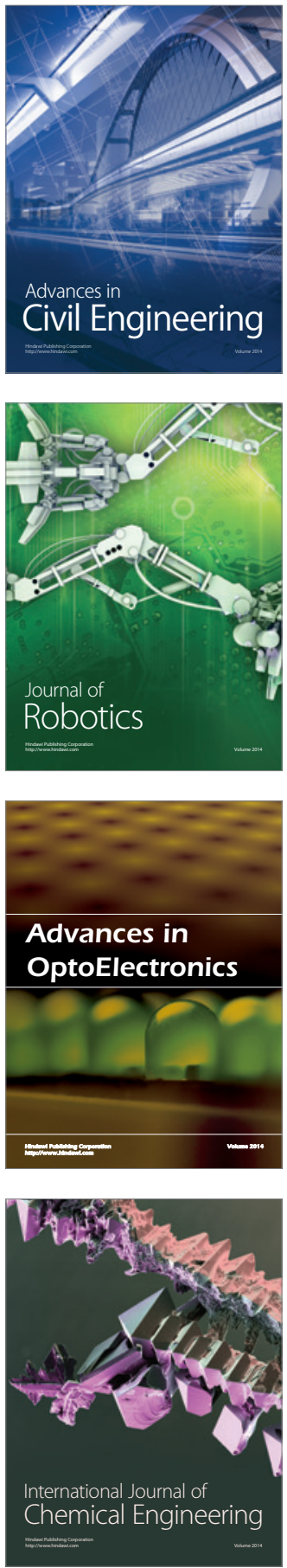

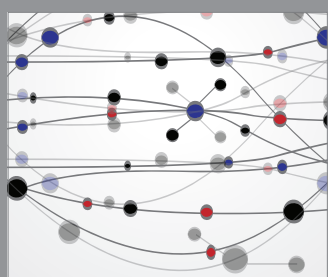

The Scientific World Journal

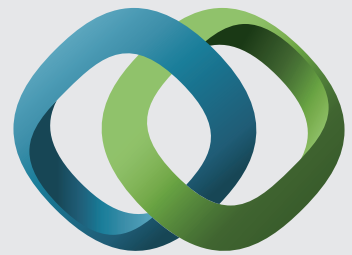

\section{Hindawi}

Submit your manuscripts at

https://www.hindawi.com
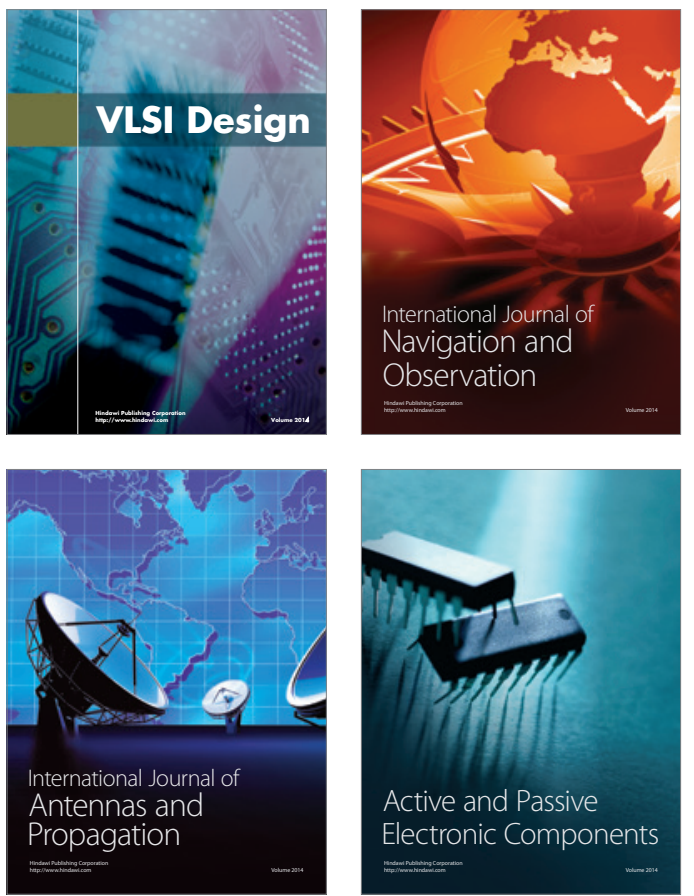
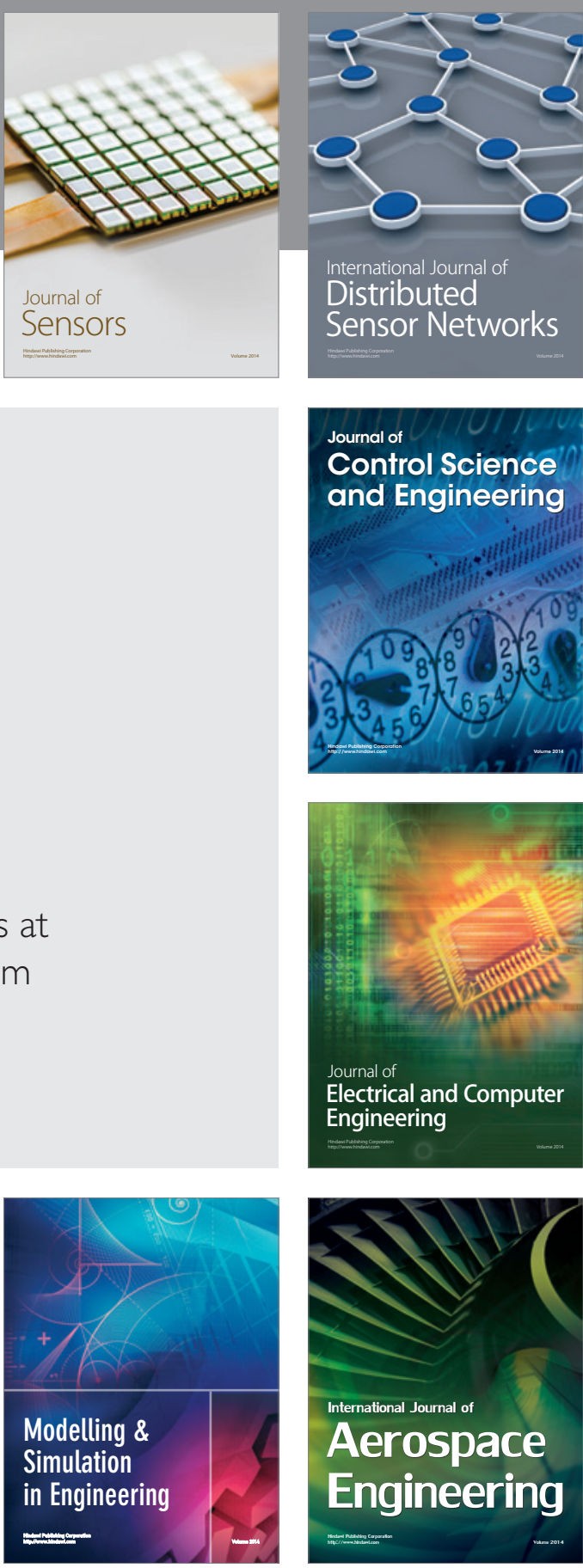

International Journal of

Distributed

Sensor Networks

$-$

Joumal of

Control Science

and Engineering
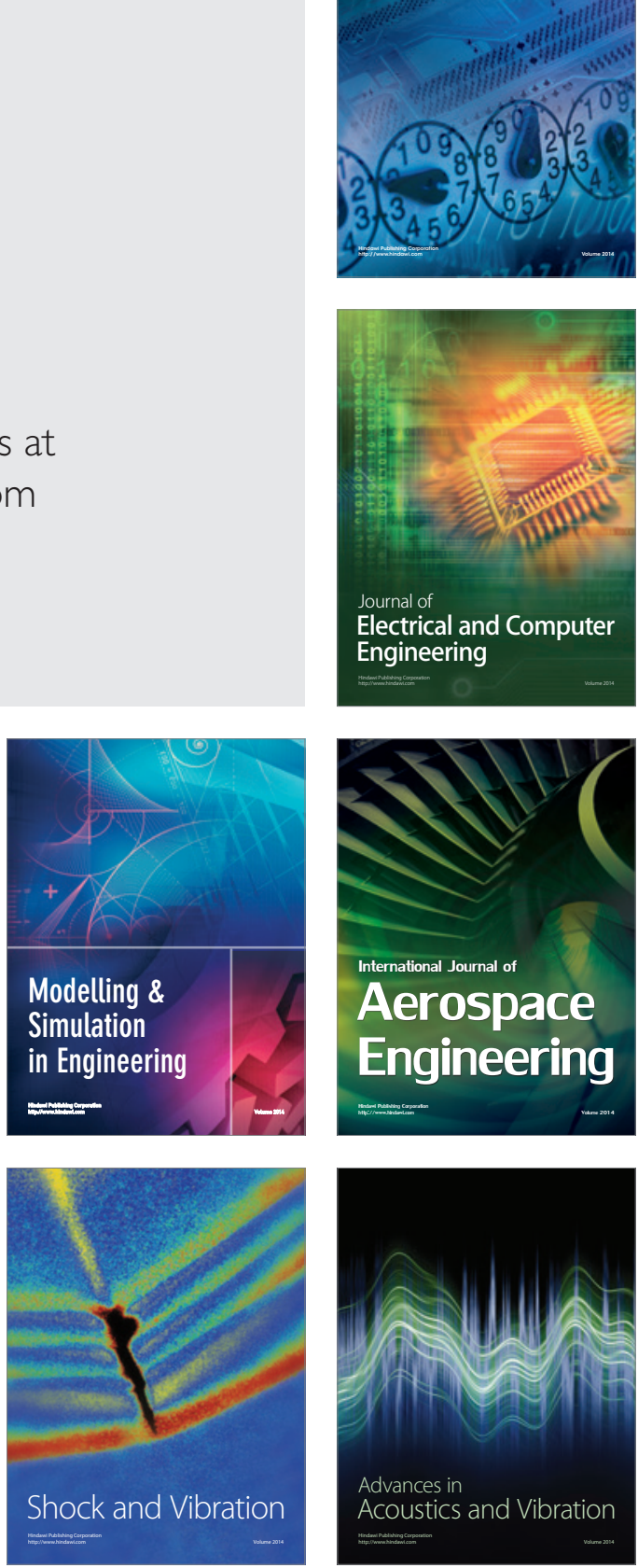